\title{
A case study testing the cavity mode model of the magnetosphere
}

\author{
D. V. Sarafopoulos \\ Department of Electrical and Computer Engineering, Demokritos University of Thrace, Xanthi, Greece
}

Received: 22 December 2004 - Revised: 6 April 2005 - Accepted: 7 April 2005 - Published: 28 July 2005

\begin{abstract}
Based on a case study we test the cavity mode model of the magnetosphere, looking for eigenfrequencies via multi-satellite and multi-instrument measurements. Geotail and ACE provide information on the interplanetary medium that dictates the input parameters of the system; the four Cluster satellites monitor the magnetopause surface waves; the POLAR ( $\mathrm{L}=9.4)$ and LANL 97A ( $\mathrm{L}=6.6)$ satellites reveal two in-situ monochromatic field line resonances (FLRs) with $\mathrm{T}=6$ and $2.5 \mathrm{~min}$, respectively; and the IMAGE ground magnetometers demonstrate latitude dependent delays in signature arrival times, as inferred by Sarafopoulos (2004b). Similar dispersive structures showing systematic delays are also extensively scrutinized by Sarafopoulos (2005) and interpreted as tightly associated with the socalled pseudo-FLRs, which show almost the same observational characteristics with an authentic FLR. In particular for this episode, successive solar wind pressure pulses produce recurring ionosphere twin vortex Hall currents which are identified on the ground as pseudo-FLRs. The BJN ground magnetometer records the pseudo-FLR (alike with the other IMAGE station responses) associated with an intense power spectral density ranging from 8 to $12 \mathrm{~min}$ and, in addition, two discrete resonant lines with $\mathrm{T}=3.5$ and $7 \mathrm{~min}$. In this case study, even though the magnetosphere is evidently affected by a broad-band compressional wave originated upstream of the bow shock, nevertheless, we do not identify any cavity mode oscillation within the magnetosphere. We fail, also, to identify any of the cavity mode frequencies proposed by Samson (1992).
\end{abstract}

Keywords. Magnetospheric physics (Magnetosphereionosphere interactions; Solar wind-magnetosphere interactions; MHD waves and instabilities)

\section{Introduction}

In the single-fluid field line resonance (FLR) model (Southwood, 1974; Chen and Hasegawa, 1974) compressional

Correspondence to: D. V. Sarafopoulos

(sarafo@ee.duth.gr) mode magnetic waves propagating in the near-Earth magnetosphere couple into transverse magnetic standing waves on closed magnetic field lines. Each field line has its own set of discrete resonant frequencies. Toroidal resonances commonly have been observed by AMPTE/CCE (Anderson et al., 1990; Nosé et al., 1995; Engebretson et al., 1986) and ISEE 1 and 2 (Mitchell et al., 1990) in a wide range of L. Conversely, Pc5 pulsations observed by ground-based magnetometers and HF (high frequency) radar data rarely exhibit this frequency spreading (see review papers of Hughes, 1994; Glassmeier, 1995a; and Takahashi, 1998; also the works of Glassmeier, 1995b; McDiarmid and Allan, 1990; Ziesolleck and McDiarmid, 1995). A possible solution to this inconsistency was offered by Kivelson et al. (1984), who suggested that the magnetosphere may resonate with its own set of eigenfrequencies (i.e. the cavity mode model). Kivelson and Southwood $(1985,1986)$ further developed this idea, postulating that a frequency-dependent turning point and the magnetopause form the inner and outer boundaries of this cavity, respectively. The model predicts energy transport from the magnetopause to the turning point for waves that propagate with the cavity eigenfrequencies (which are determined by magnetospheric boundary conditions). The theoretical models for the cavity mode include a simple box geometry with perfectly reflecting boundaries (Kivelson and Southwood, 1986), a rectangular wave guide (Samson et al., 1992), a cylindrical magnetosphere (Allan et al., 1986) or a dipole magnetosphere (Lee and Lysak, 1989). The differences in the magnetospheric geometry lead to different mode structures.

An important factor to be considered, in addition to cavity geometry, is loss of the fast-mode energy by various mechanisms (for instance, conversion into the shear Alfvén waves, Joule dissipation caused by finite ionospheric conductivity, tailward escape, and loss into the solar wind through the magnetopause). Obviously, loss of fast-mode energy through these mechanisms reduces the $Q$ value of the magnetospheric cavity mode. Whether one can detect the compressional eigenmodes as narrow-band oscillations should depend critically on how quickly the fast-mode energy is lost out of the cavity. No numerical studies have made a comprehensive assessment of the energy loss mechanisms, so the debate continues about whether the cavity mode can be experimentally 
detected (Takahashi, 1998).

Another factor that must be considered in trying to identify the magnetospheric cavity/waveguide mode, and in distinguishing between forced and standing compressional oscillations of the magnetospheric cavity, requires a careful inspection of the solar wind variations that impinge on the magnetopause and the mode structure of magnetic field perturbations in the magnetosphere. A multiharmonic cavity mode may be excited by a broad-band solar wind pressure wave. According to computer simulations, assuming perfectly reflecting boundaries (e.g. Lee and Lysak, 1991a), not only the cavity mode but also the multiharmonic toroidal resonances (Alfvén continuum) are excited by the impulsive source. In contrast, when the solar wind pressure (or some other external quantity) changes periodically in a sinusoidal manner, the magnetospheric field will oscillate with the same periodicity. According to numerical simulations (Lee and Lysak, 1991b), the compressional oscillations can couple to toroidal-mode standing Alfvén waves at the locations where the driver frequency matches the local toroidal-mode Alfvén frequency.

Harrold and Samson (1992) and Samson et al. (1992) proposed that pulsations with discrete frequencies of 1.3, 1.9, 2.6, 3.4, and $4.2 \mathrm{mHz}$ (the so-called CMS frequencies, after the cavity mode model of Samson et al., 1991) observed by high-latitude radar arise from a waveguide mode on the flankside of the magnetosphere. The latter motivated several studies that searched for pulsations having these frequencies, while Samson et al. (1992) and Walker et al. (1992) reported that these frequencies vary little and do not depend on the geomagnetic condition.

There are studies in support of the presence of CMS frequencies at different latitudes and from different instruments. Shimazu et al. (1995) report multipoint observations of a Pc5 pulsation having discrete frequencies of $3.3,4.7,5.9$, and $7.1 \mathrm{mHz}$ following a minor increase in the solar wind dynamic pressure, at latitudes ranging from the auroral zone to the geomagnetic equator. Provan and Yeoman (1997) use the Wick radars $(\mathrm{L}=4-6.5)$ and find some of the CMS frequencies at mid-latitude. An additional report of the CMS frequencies comes from magnetometer observations at a low latitude ( $\mathrm{L}=1.6)$ station (Francia and Villante, 1997). Further support for the CMS frequencies was provided by Fenrich et al. (1995), who studied 12 FLR events with SuperDARN data. Their results indicated that the CMS frequencies were prevalent at all magnetic local times. Ziesolleck and McDiarmid (1995) conducted a statistical study, based on the CANOPUS magnetometer data $(\mathrm{L}=4.2-12.3)$, and in contrast to the event studies listed above, their results demonstrated that while FLRs occur with the same frequency at all latitudes, they do not preferentially occur at the CMS frequencies. In fact, Ziesolleck and McDiarmid (1995) proposed three new sets of discrete frequencies which might be more prevalent than the CMS frequencies. More recent Pc5 ground pulsation studies have also addressed the subject of stable, repeatable, discrete frequencies. Mathie et al. (1999) found that the CMS frequencies were prominent in their 137 pulsation events but were unable to confirm that the frequen- cies were stable. Chisham and Orr (1997) found no evidence of the CMS frequencies in their 129 events but did find a set of recurring frequencies that were consistent with one of the frequency sets proposed by Ziesolleck and McDiarmid (1995). Usually pulsations are observed under a large range of solar wind conditions, a large set of magnetospheric configurations, a variety of boundary conditions applied to the waveguide and consequently, the eigenfrequencies may change accordingly (Baker et al., 2003).

Finally, we note that there is a class of Pc5 pulsations that are directly driven by periodic disturbances in the solar wind. Korotova and Sibeck (1995) and Matsuoka et al. (1995) used ground and geosynchronous (or near-geosynchronous) satellite observations, along with a solar wind monitor and reported Pc5-band pulsations that are associated with similar periodic changes in the dynamic pressure of the solar wind. A similar but more persistent solar-wind-driven pulsation event in the tail lobe is reported by Sarafopoulos (1995), using IMP-8 observations. Because of the simultaneous solar wind measurements of the dynamic pressure by the ISEE 3 spacecraft, there is little doubt that the lobe oscillation is a direct response to the solar wind oscillation. The Sarafopoulos event lasted 2-3 $\mathrm{h}$ and had a frequency of $1.7 \mathrm{mHz}$, which is quite close to one of the CMS frequencies. The pulsation reported by Korotova and Sibeck (1995) had a frequency of $2.2 \mathrm{mHz}$, and those reported by Matsuoka et al. (1995) also had a median frequency of $2.2 \mathrm{mHz}$. From these examples it is clear that disturbances in the solar wind can be a direct cause of low-frequency pulsations observed on the ground and that wave guide modes are not necessarily the only cause of Pc5 pulsations. The question is how often these solarwind-induced magnetic pulsations contribute to the pulsations observed on the ground by radar or magnetometers (Takahashi, 1998). It is of great importance to examine the state of the solar wind at times when magnetic pulsations are observed on the ground, and this is the decisive element for this work case study. Not only does solar wind directly drive magnetospheric pulsations, but it should also control the frequency of magnetospheric resonances. As for the waveguide mode, we note that the distance of the outer boundary will become smaller for a larger solar wind dynamic pressure or a larger southward component of the interplanetary magnetic field.

Sarafopoulos (2004a) provided conclusive observational evidence demonstrating that a solar wind pressure pulse produces a twin-vortex system of ionospheric currents, while a stepwise pressure increase/decrease creates a single vortex structure, at high-latitude ground magnetograms. Sarafopoulos (2004b) observed latitude-dependent delays in signature arrival times at the dawnside ground magnetograms and demonstrated that these structures are directly dictated by successive exo-magnetosphere pressure pulses applied along the magnetopause. He established (using ground and satellite data, together with results from the Tsyganenko T96 model of the magnetosphere) the conditions under which a compression wave travelling tailward along the magnetopause surface will produce poleward moving signatures in ground 


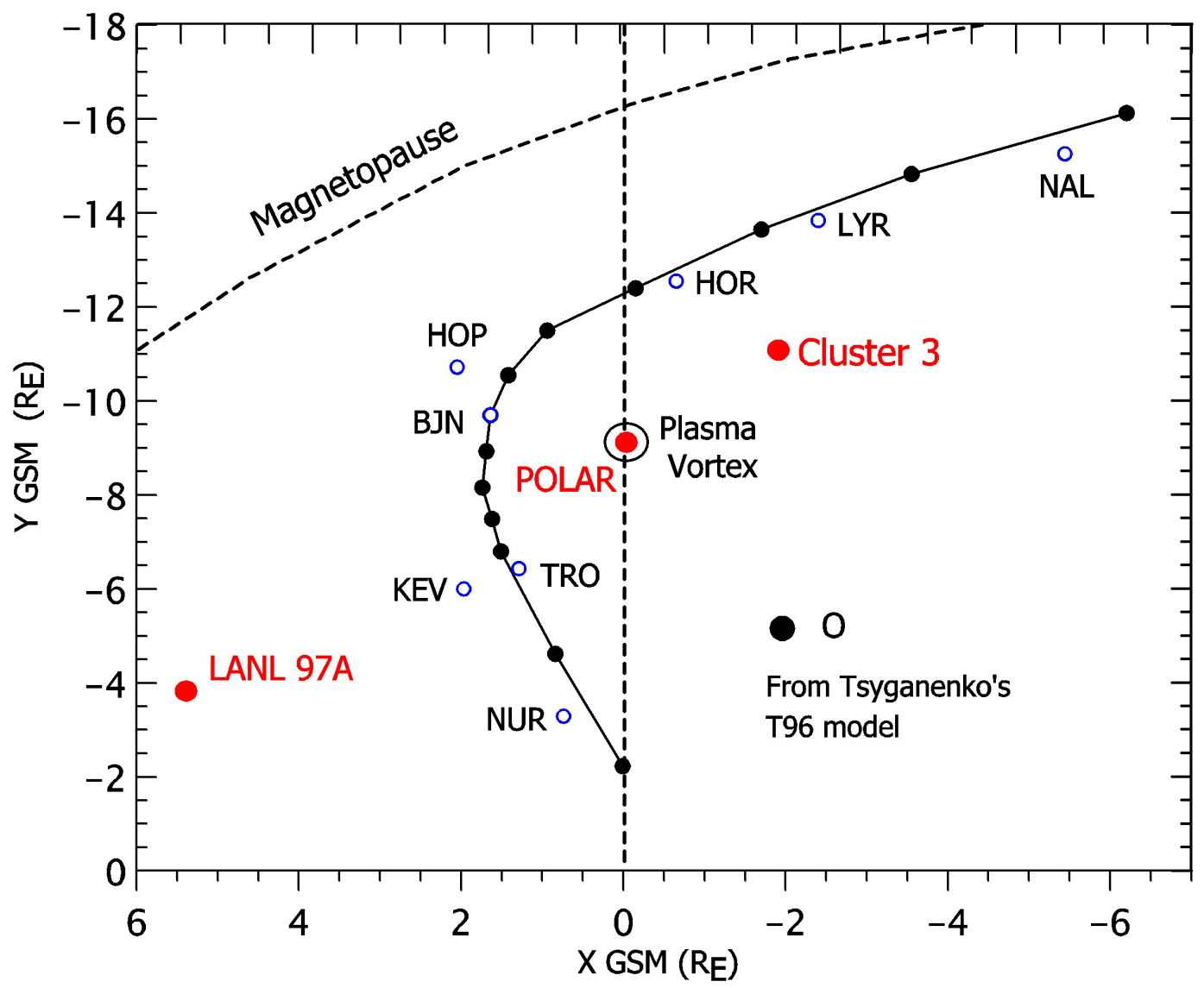

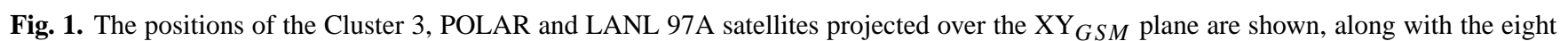
conjugate points of the ground stations NAL, LYR, HOR, HOP, BJN, TRO, KEV and NUR over the equatorial plane (open circles). The solid circle symbols correspond to a hypothetic chain of ground stations having progressively increasing latitudes, while their longitude is $19.2^{\circ}$ (i.e. the $\mathrm{BJN}$ longitude).

magnetograms. This phenomenon, characterized by ground dispersive structures, produces waveforms out of phase, and changes the pulsation polarization sense, much like the FLR mechanism (Sarafopoulos, 2005). The event under study is associated with twin-vortex ionosphere structures and can be termed as a pseudo-FLR event. Given that many works in the past that engaged with ground Pc5 pulsations do not incorporate the solar wind conditions, it is probably inevitable that many events selected as FLRs are, in fact, repetitive twinvortex structures. Under the Sarafopoulos $(2004 b, 2005)$ perspective the observation that all the auroral ground stations show the same frequency, which is associated with successive twin-vortex current systems imposed by a magnetopause surface wave, has nothing to do with the cavity mode. The latter is the subject for this study, which further extends this author thinking. Despite the large amount of work that has been conducted in the past, the issue concerning the magnetospheric cavity mode eigenfrequencies remains controversial. This work tests the cavity mode model using a case study.

\section{Observations}

This work deals with a case study of day 181, 2001, that incorporates measurements obtained (a) in the solar wind plasma regime (Geotail and ACE satellites), (b) at the magnetopause surface (four Cluster satellites), (c) well-inside the magnetosphere (POLAR and LANL satellites), and (d) on the Earth's surface (through the IMAGE array stations corresponding to the dawn sector plasma sheet). As a result, first, we have knowledge of the exo-magnetosphere conditions determining the energy input for the system. Second, we are certain that the magnetopause surface actually oscillates and propagates inward compressional waves which are associated with significant power spectral density (PSD). Third, the in-situ measurements within the magnetosphere give us the opportunity to probe the local FLRs, or the supposed cavity/waveguide mode oscillations. Finally, the ground magnetograms are of crucial importance to trace the ionosphere response and classify the ground signatures according to their spatial and temporal structures. The same time interval was studied, in part, by Sarafopoulos (2004b); and Fig. 1 presents the result from the mapping process of the IMAGE array ground stations (i.e. NAL, LYR, HOR, HOP, BJN, TRO, 
KEV and NUR) via Tsyganenko's T96 model (Tsyganenko, 1995, 1996) over the equatorial plane, something which is shown previously by Sarafopoulos (2004b, in his Fig. 10). The open circle symbols correspond to the real ground station coordinates, whereas the solid circles correspond to a hypothetic chain of ground stations having the same geographic longitude of $19.2^{\circ}$ (i.e. the BJN station longitude). Eventually, the integration of all the available information will enable us to search in depth whether a cavity/waveguide excitation mode in the Pc5 band of frequencies has actually taken place.

\subsection{Response of the POLAR satellite}

We examine the Pc5 frequency pulsations of plasma density and magnetic field that occur during a pass of the POLAR satellite along the dawn meridian and close to the geomagnetic equator; an interval of two hours (03:45 to 05:45 UT) of day 181, 2001, is under study. At 04:50 UT the POLAR satellite was positioned (see Fig. 1) at $(\mathrm{X}, \mathrm{Y}, \mathrm{Z})_{G S M}=(0.107$, $-9.16,2.04) R_{E}$. We use plasma data from the Thermal Ion Dynamics Experiment (TIDE), vector magnetic field data from the Magnetic Field Experiment (MFE), and electric field $\left(\mathrm{E}_{x y}\right.$ and $\left.\mathrm{E}_{z}\right)$ measurements from the Electric Field Instrument (EFI). Figure 2 clearly shows 10 variation cycles (pulsations) observed by POLAR from 04:25 to 05:20 UT. The periodicity $\mathrm{T}$ is exactly $6 \mathrm{~min}$ around the time of the largest amplitude waves. The plasma ion density (top panel) and velocity $\mathrm{V}_{x}$ and $\mathrm{V}_{y}$ components (second and third panels, respectively) are very well modulated by the wave. The strongest component of the magnetic field oscillation is the $\mathrm{B}_{x}$ (fourth panel), while the $\mathrm{B}_{y}$ and $\mathrm{B}_{z}$ components are weak and somewhat irregular (not shown here). Instead of presenting $\mathrm{B}_{y}$ and $\mathrm{B}_{z}$, we prefer to show the compressional and transverse components of the GSM vector magnetic field that is transformed to a field-aligned coordinate system in which $\hat{\mathbf{c}}$ is the unit vector along the average field for the interval of Fig. 2; $\hat{\mathbf{i}}=\hat{\mathbf{e}} \mathbf{x} \hat{\mathbf{c}}$, where $\hat{\mathbf{e}}$ is the duskward unit vector, and $\mathbf{j}=\hat{\mathbf{c}} \mathbf{x} \hat{\mathbf{i}}$. The new components are the transverse components $\mathrm{B}_{i}$ and $\mathrm{B}_{j}$, and the compressional component $\mathrm{B}_{c}$ (bottom three panels). The $\mathrm{B}_{i}$ is almost identical to the $\mathrm{B}_{x}$ shown in the fourth panel. In this POLAR position, along the dawn meridian, the $\mathrm{X}$ direction is azimuthal, while the $\mathrm{Y}$ direction is nearly radial. Therefore, the pulsations under study show clearly a toroidal mode of waves.

Figure 3 keeps a close watch on two variation cycles (marked as A and B along the ion density profile, top panel), which are representative of the whole event. The electric field shows a strong oscillation in the $\mathrm{E}_{z}$ component (bottom panel) and a weaker one over the XY plane (fifth panel). The dominant variation for the electric field component $\mathrm{E}_{z}$ leads the variation for the dominant magnetic field component $\mathrm{B}_{x}$ (fourth panel) by $90^{\circ}$. This phase relation implies a standing transverse wave at the fundamental mode. In particular, it shows the same frequency, at almost the same site, as in the case studied by Mitchell et al. (1990) using the ISEE 1 and 2 satellites. It is worth noticing that there is a quantitative agreement between the velocities derived from the plasma experiment and the observed electric field: For instance, given that the $\mathrm{V}_{y}$ prevails over the $\mathrm{V}_{x}$, then $\mathrm{E}_{x} \approx \mathrm{E}_{x y} \approx \mathrm{V}_{y} \mathrm{~B}_{z} \approx 30 \mathrm{~km} \cdot \mathrm{s}^{-1} * 32 \mathrm{nT} \approx 1 \mathrm{mV} / \mathrm{m}$, which is the directly measured value via the EFI instrument. Given that for standing Alfvén waves the density remains constant, we conclude that the periodic density variations have to be associated with the compressional $\mathrm{B}_{c}$ component of the magnetic field (Fig. 2, bottom panel).

The hodogram for the velocity components $\mathrm{V}_{x}$ and $\mathrm{V}_{y}$, as it results from a careful inspection of the two representatively shown variation cycles in Fig. 3, is schematically drawn in Fig. 4. Each variation cycle corresponds to a plasma vortex with an average diameter of $\sim 0.5 \mathrm{R}_{E}$. The letters marked along the $\mathrm{V}_{x}$ and $\mathrm{V}_{y}$ traces in Fig. 3 correspond to those used in the hodogram of Fig. 4. The polarization sense is righthanded $(\mathrm{RH})$, and each vortex over the XY plane is associated with a converging electric field topology marked with arrows across the circular plasma path in Fig. 4. The electric field has the value $\mathrm{E}=-\mathrm{U}_{x y} \times \mathrm{B}_{z}$, and as it applies over the ionosphere Joule dissipation takes place. If this plasma sheet vortex is projected over the ionosphere and is scaled down $\sim 30$ times (Glassmeier et al., 1999), then it is probable that the ionosphere currents associated with this vortex will not be detectable by the ground-based magnetometers (because of the spatial integration effect). In summary, Fig. 2 shows ten (out of more than twenty) successive vortex structures associated with almost monochromatic oscillations of $\mathrm{B}_{x}$ with $\mathrm{T}=6 \mathrm{~min}$, which, we believe, to be the resonant ringing of the medium at this $\mathrm{L}$ shell region. This pulsation event occurs with very low geomagnetic activity and the magnetopause surface probably constitutes the broad-band energy source for the waves under investigation.

\subsection{Solar wind conditions obtained by Geotail and ACE}

In parallel to the repetitive plasma vortices seen by POLAR, it is of great importance to look at the solar wind conditions. Although for this event conditions are essentially analyzed in a previous work (Sarafopoulos, 2004b; Figs. 1, 4 and 5) it is useful, however, to present here the main solar wind variations that influence the magnetosphere. Additionally, we incorporate, for the first time, the most critical parameter which is the solar wind dynamic pressure. We mention that Geotail and ACE were located at $(\mathrm{X}, \mathrm{Y}, \mathrm{Z})_{G S E}=(15.27,2.43$, 1.66) and $(247.8,20.4,14.3) \mathrm{R}_{E}$, respectively, at 05:00 UT. Figure 5 shows, from top to bottom: (a) the three major IMF amplitude depressions recorded by Geotail, marked with the capital letters A, B and C; (b) the solar wind ion pressure (in $\mathrm{nPa}$ ) and density (in $\mathrm{cm}^{-3}$ ) measured by Geotail; (c) the ACE satellite magnetic field amplitude shifted in time 71 min to match the Geotail data, and (d) the Cluster $3 \mathrm{~B}_{y}$ component of the magnetic field showing three intermittent exits of the satellite from the plasma mantle into the magnetosheath. The negative value of $\mathrm{B}_{y}$ in the magnetosheath was inferred by the IMF measured on board Geotail (not shown here). Although the resolution time of the Geotail CPI/SWA 

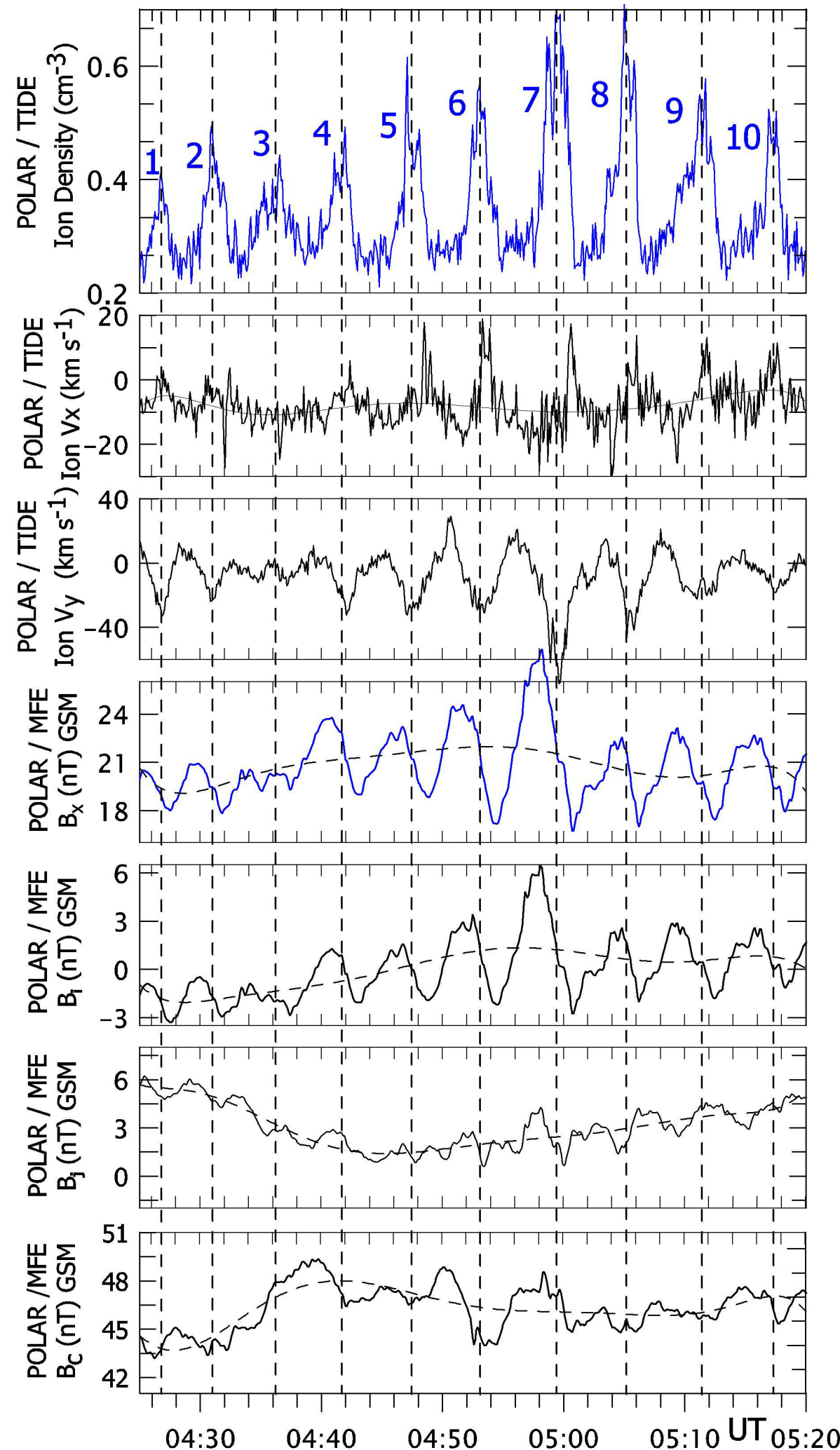

Fig. 2. Measurements from the POLAR satellite: (a) ten variation cycles of the ion density (in $\mathrm{cm}^{-3}$ ), (b) ion $\mathrm{V}_{x}$ and $\mathrm{V}_{y}$ velocities (in $\mathrm{km} \mathrm{s}^{-1}$ ), (c) magnetic field $\mathrm{B}_{x}$ component (in nTs), and (d) the transverse $\mathrm{B}_{i}$ and $\mathrm{B}_{j}$ and compressional $\mathrm{B}_{c}$ components (bottom three panels) of the GSM vector magnetic field, which is transformed to a field-aligned coordinate system in which $\hat{\mathbf{c}}$ is the unit vector along the average field for the shown interval, $\hat{\mathbf{i}}=\hat{\mathbf{e}} \mathbf{x} \hat{\mathbf{c}}$, where $\hat{\mathbf{e}}$ is the duskward unit vector, and $\mathbf{j}=\hat{\mathbf{c}} \mathbf{x} \hat{\mathbf{i}}$. The toroidal character of waves is apparent. 


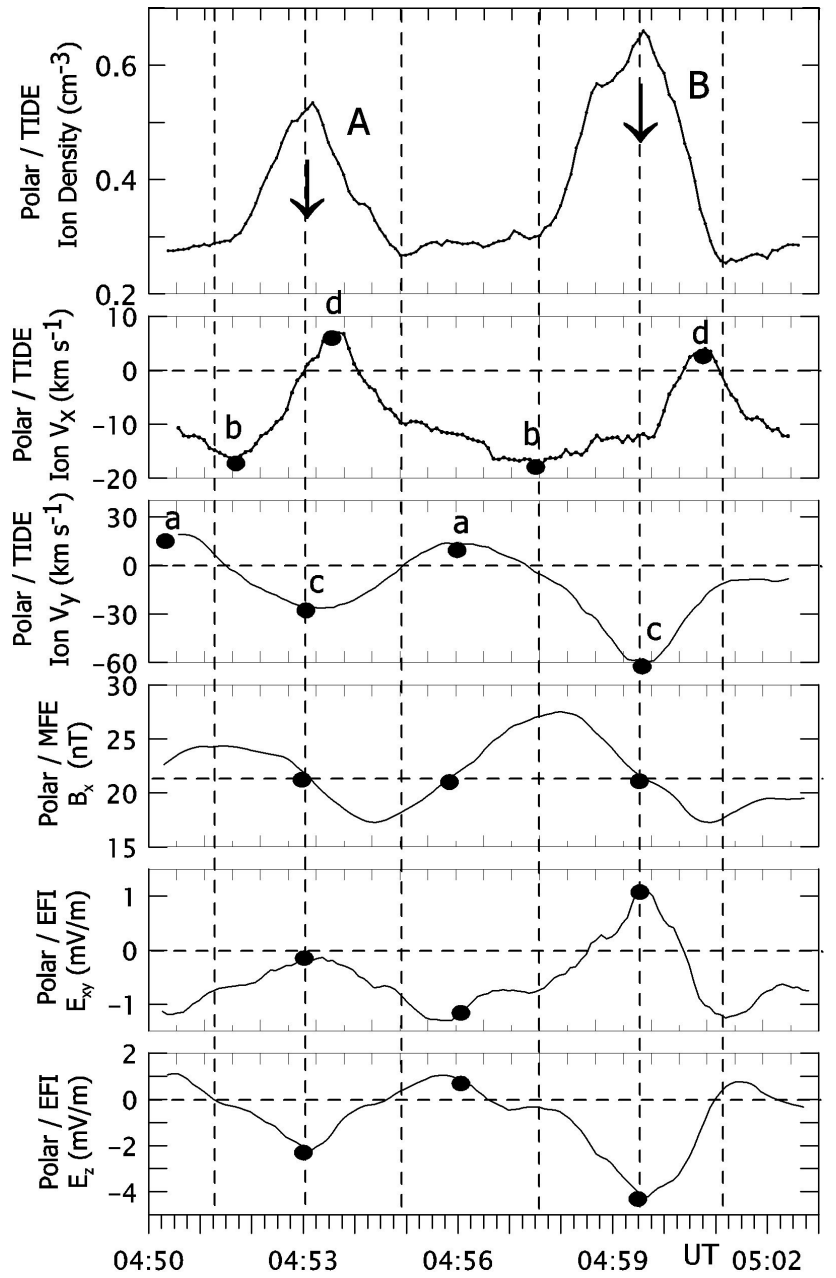

Fig. 3. Two variation cycles of POLAR ion density (top panel) associated with the ion velocities $\mathrm{V}_{x}$ and $\mathrm{V}_{y}$ (second and third panels), the magnetic field $\mathrm{B}_{x}$ toroidal wave (fourth panel) and the electric field $\mathrm{E}_{x y}$ and $\mathrm{E}_{z}$ components (in $\mathrm{mV} \mathrm{m}^{-1}$ ).

plasma experiment is $\sim 51 \mathrm{~s}$, nevertheless, we are certain that three major solar wind pressure increases are anticorrelated to the IMF amplitude depressions. Then, these pressure variations are applied over the magnetosphere, forcing Cluster 3 to exit three times out of the magnetosphere cavity (bottom panel). Cluster 3 was located at $(\mathrm{X}, \mathrm{Y}, \mathrm{Z})_{G S E}=(-1.9$, $-10.67,8.56) \mathrm{R}_{E}$, at 05:00 UT (Fig. 1). The dashed line in the top panel results as a polynomial fitting of order four. The ACE magnetic field trace (fourth panel) confirms that the three characteristic variations marked as $\mathrm{A}, \mathrm{B}$ and $\mathrm{C}$ are inherent to the solar wind plasma regime.

\subsection{Response of the LANL 97A satellite}

The geographic longitude for the geostationary satellite LANL 97A is $70.2^{\circ}$, and at 05:00 UT it is positioned at $(\mathrm{X}, \mathrm{Y})=(5.42,-3.77) \mathrm{R}_{E}$. This dayside position (Fig. 1) allows 97A to be affected earlier than the POLAR satellite from the three solar wind pressure pulses (Sarafopoulos, 2004b). Figure 6, from top to bottom, shows (a) the

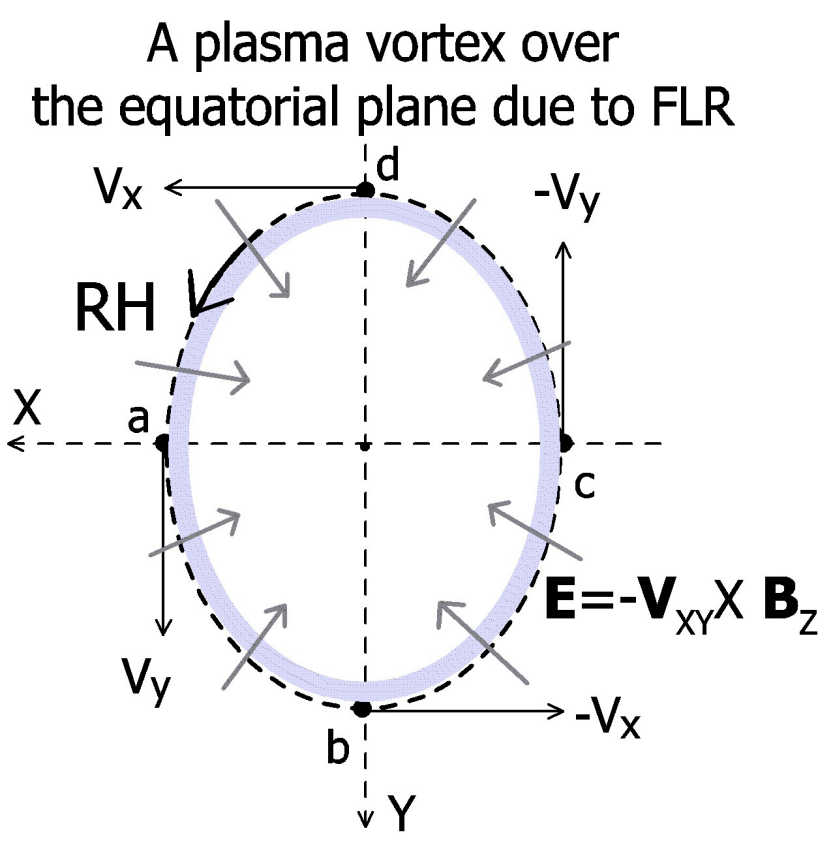

Fig. 4. One plasma vortex (out of ten shown in Fig. 1) is schematically shown as the hodogram of $\mathrm{V}_{x}$ and $\mathrm{V}_{y}$ taken from Fig. 3. The letters a-d show a velocity vector reorientation in steps of $90^{\circ}$.

LYR station X-component magnetogram with three distinct decreases marked with the capital letters A, B and C; (b) the 97A differential fluxes for two energetic proton channels with energies 50-75 and 75-113 keV (second panel, thin lines); (c) the magnetic field compressional component $\mathrm{B}_{c}$ measured by POLAR; and (d) the POLAR ion density profile. The dotted curves in Fig. 6 correspond to low-pass filtered data with cutoff frequencies 2 and $2.4 \mathrm{mHz}$ for the $\mathrm{PO}$ LAR (third panel) and LANL 97A data, respectively. The applied frequency cutoffs are dictated by the power spectra, which are presented later on. These filtered energetic proton fluxes of 97A, as well as the magnetic field component $\mathrm{B}_{c}$ of POLAR, clearly show three major increases that are obviously associated with the compressions A, B and C. In this way, at the LANL 97A site, it is demonstrated that a wave with a periodicity of $\sim 2.5 \mathrm{~min}$ is superimposed over the three major decreases caused by the solar wind pressure pulses. The thin-dashed vertical lines (second panel) emphasize the high frequency wave with $\mathrm{T}=2.5 \mathrm{~min}$. The three thick-dashed vertical lines mark the occurrence times of pressure pulses at different sites. Although the POLAR magnetic field pulsations are largely non-compressive, with a dominant $\mathrm{B}_{x}$ oscillation, however, we recognize a weak compressional component with $\Delta \mathrm{B} / \mathrm{B} \cong 0.045$, which is modulated by the three magnetosphere compressions. Much like the fact that the POLAR ion density profile shows a single unique oscillation frequency, which is interpreted as the manifestation of the local FLR phenomenon at $\mathrm{C} \cong 9.4$, the 97A energetic particle discrete and stable periodicity of $\sim 2.5$ min reflects the local FLR at $\mathrm{L}=6.6$. It seems that the 97A particle fluxes respond passively to the magnetic field structure oscillations. 
We assume that the currents associated with the FLRs (transverse oscillations) propagate along the magnetic field lines, whereas the wave pressure information (variations A, B and C) at $\mathrm{L}=6.6$ and 9.4 is transmitted by fast mode waves.

The POLAR FLR oscillations persist before and after the interval confined between the three marked solar wind pressure variations. Nevertheless, the POLAR ion density wave increases its amplitude within this interval, an indication that the fast mode wave energy is partly consumed via local FLRs.

\subsection{Response of the four Cluster satellites}

The Cluster 3 spacecraft, at 05:00 UT, was positioned at (X, $\mathrm{Y}, \mathrm{Z})_{G S E}=(-1.9,-10.67,8.56) \mathrm{R}_{E}$, and Cluster 1, 2, and 4, relatively to Cluster 3 , were positioned at

$(\Delta \mathrm{X} 1, \Delta \mathrm{Y} 1, \Delta \mathrm{Z} 1)=(323,-1918,1907) \mathrm{km}$,

$(\Delta \mathrm{X} 2, \Delta \mathrm{Y} 2, \Delta \mathrm{Z} 3)=(-1550,-2715,1542) \mathrm{km}$, and

$(\Delta \mathrm{X} 4, \Delta \mathrm{Y} 4, \Delta \mathrm{Z} 4)=(-1688,-4357,389) \mathrm{km}$.

Cluster 3 remains at the high latitude boundary layer (HLBL) or plasma mantle, and its position (being the innermost of all the four satellites) is marked in Fig. 7 over the YZ crosssectional plane. The other Cluster positions, relatively to Cluster 3, are shown using the axis $\Delta \mathrm{Y}$ and $\Delta \mathrm{Z}$. The circular thick-dashed line represents an ideal circular magnetopause surface, and Cluster 3 is contained within this boundary. When each of the three solar wind pressure increases reduces the magnetotail diameter, Cluster 3 crosses the boundary and exits into the magnetosheath. The latter is evident from the bottom panel of Fig. 5 (see also the Fig. 5 in the work of Sarafopoulos, 2004b) showing the Cluster $3 \mathrm{~B}_{y}$ magnetic field component measured by the FGM experiment (Balogh et al., 1997). The negative values along the $\mathrm{B}_{y}$ trace represent the magnetic field lines that are draped around the magnetopause, given that the IMF has an intense negative $\mathrm{B}_{y}$ component about $-3 \mathrm{nT}$. Therefore, the three successive exits of Cluster 3 are caused by three intense compressions applied over the magnetopause. The resulted surface wave propagates tailward with an estimated velocity $\sim 210 \mathrm{~km} \cdot \mathrm{s}^{-1}$, a surface wavelength $\sim 16 \mathrm{R}_{E}$, and an azimuthal wave number $\mathrm{m} \cong 6$ (Sarafopoulos, 2004b).

After the just presented "first time interval" the situation seems to be radically different. The amplitude of the magnetopause surface wave is apparently significantly reduced, and consequently, Cluster 3 remains permanently inside the magnetopause. In contrast, the other three Cluster satellites remain essentially in the magnetosheath plasma regime $\left(\mathrm{B}_{y}<0\right)$ and quasi-periodically enter into the magnetosphere $\left(\mathrm{B}_{y}>0\right)$. The latter is clearly demonstrated via Fig. 8, showing the $\mathrm{B}_{y}$ responses for all the four Cluster satellites, in four successive magnetotail expansions/contractions. This "second time interval" of Fig. 8 is not associated with an intense ULF activity visible along the IMF amplitude trace (first panel) or the solar wind ion pressure (second panel), in contrast to the situation of the preceding period of Fig. 5. Actually, the Geotail time series, which are time-shifted $13 \mathrm{~min}$ to match the Cluster data, do not show any repetitive varia-

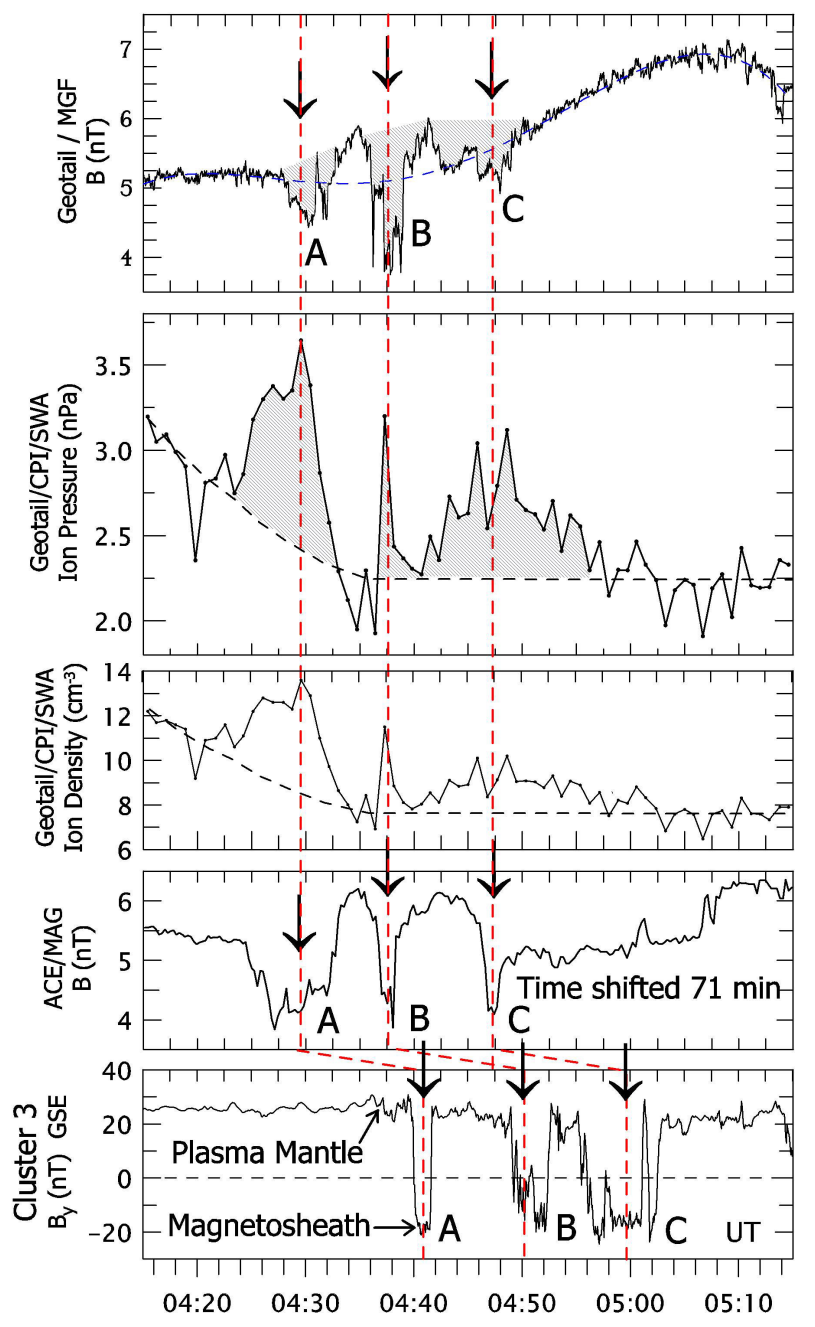

Fig. 5. From top to bottom: (a) the IMF amplitude (in nT) recorded by Geotail, showing three major depressions marked with the capital letters A, B and C; (b) the solar wind ion pressure (in $\mathrm{nPa}$ ) and density (in $\mathrm{cm}^{-3}$ ) measured by Geotail; (c) the ACE satellite magnetic field amplitude shifted in time $71 \mathrm{~min}$ to match the Geotail data, and (d) the Cluster $3 \mathrm{~B}_{y}$ component of the magnetic field showing three intermittent exits of the satellite from the plasma mantle into the magnetosheath.

tion which could be associated with the four variation cycles marked with the numbers $1-4$ along the Cluster $4 \mathrm{~B}_{y}$ trace, or the five variation cycles marked with the numbers $1-5$ along the POLAR ion density trace (third panel). In this case, we are almost certain that the surface wave is not directly driven by the solar wind conditions, and that KHI may be the specific mechanism producing it.

\section{Discussion}

\subsection{Simultaneous pseudo- and authentic-FLRs}

In Fig. 9 the power spectral densities (PSDs) are computed for a few time series obtained from the POLAR and LANL 




Fig. 6. From top to bottom: X-component magnetogram from the ground station LYR; differential fluxes from the two energetic proton LANL 97A channels (50-75 and 75-113 keV, thin lines); and compressional magnetic field component and ion density from the POLAR satellite. The dotted-thick lines represent low-pass filtered data, while the capital letters A, B and C mark the occurrence times for the three solar wind pressure pulses, which, in turn, modulate the 97 A fluxes and the POLAR total magnetic field trace. The 97A fluxes are clearly modulated by a wave of period $\mathrm{T} \cong 2.5 \mathrm{~min}$.

97A satellites and the ground station LYR. The purpose is to determine the underlying excitation mechanisms for the identified periodic variations. All four spectra shown are computed for the interval 04:30-05:20 UT, corresponding exactly to that shown in Fig. 6, and having the prominent feature that three successive exo-magnetosphere compressions occur within it. The X-component magnetogram from the LYR station (first panel) shows wave activity confined be- tween $\mathrm{T}=5.5$ and $12 \mathrm{~min}$; with the assumption that significant frequencies are those lying above the half-maximum PSD level. At this moment we mention, again, that the $X$ component magnetograms of all the IMAGE stations demonstrate, for the case under study, the latitude dependent dispersive structures shown by Sarafopoulos (2004b, Fig. 1) and are interpreted as the manifestation of pseudo-FLRs, which are associated with the formation of twin-vortex 
ionosphere current structures, a notion well-developed recently by Sarafopoulos (2005). In addition, it was established by Sarafopoulos (2004a) that each solar wind pressure pulse produces a twin-vortex system according to his multisatellite observations. Therefore, the LYR station wave activity (Fig. 9) probably reflects the direct influence of the three solar wind pressure pulses associated with the pseudo-FLRs.

The LANL 97A energetic proton fluxes, for the energy channel 75-113 keV (Fig. 9, second panel), show two distinct periodicities: one at $\mathrm{T} \cong 2.5 \mathrm{~min}$, which most probably is due to the local FLR and has a monochromatic character, and a second at around $8.5 \mathrm{~min}$, which is due to the solar wind pressure pulses and has a more broad-band character. The ion density PSD of POLAR (Fig. 9, third panel) shows a unique dominant periodicity at $\mathrm{T}=6 \mathrm{~min}$ originated by the local FLR mechanism. Finally, the PSD computed from the POLAR magnetic field strength (fourth panel) shows two periodicities: one at $\mathrm{T}=6 \mathrm{~min}$, which is the already identified local FLR oscillation, and a second at $\mathrm{T} \cong 10 \mathrm{~min}$, which is most probably caused by the solar wind pressure variation. Therefore, we believe that the differentiation between authenticand pseudo-FLRs is very clear. It is demonstrated, with insitu measurements, that genuine FLRs are actually excited as monochromatic waves at two widely separated sites within the magnetospheric cavity. In contrast, the pseudo-FLRs detected by ground magnetometers or satellites (and marked in Fig. 9) are more broad-band in their character and probably are driven by the three exo-magnetosphere successive compressions.

\subsection{Energy source for the FLR and non-FLR oscillations}

To understand better the interaction among the solar wind variations, the magnetopause motion and the ionosphere currents, we compute a few more power spectra. We divide the whole interval under study into two subintervals: the first one that essentially corresponds to the occurrence time of the three solar wind pulses (Fig. 10, interval from 04:20 to $05: 10 \mathrm{UT}$, in which the Geotail data are time-shifted $13 \mathrm{~min}$ to match the magnetosphere data), and the second one, in which, although the magnetopause surface quasiperiodically oscillates as well (Fig. 11, interval from 05:03 to 05:32 UT, that is exactly that presented in Fig. 8), the solar wind parameters do not show any significant variation. First, we compute for both intervals the PSDs of the IMF amplitude (Fig. 10, second panel and Fig. 11, top panel), given that these high resolution measurements are anticorrelated to the solar wind pressure (Fig. 5). Actually, the computed PSD for the solar wind ion pressure (Fig. 10, first panel) shows the same two major peaks as the IMF, even though the plasma data are of low resolution. The result is that (a) the input energy of the solar wind has actually a broad-band character ( $\mathrm{T}=5-12 \mathrm{~min})$, and (b) the input energy is much smaller for the second interval, given that both of the computed IMF PSDs are displayed in scale. The high latitude ground station LYR (Fig. 9) has essentially a similar power spectrum as the IMF amplitude in Fig. 10. During both subintervals the mag-

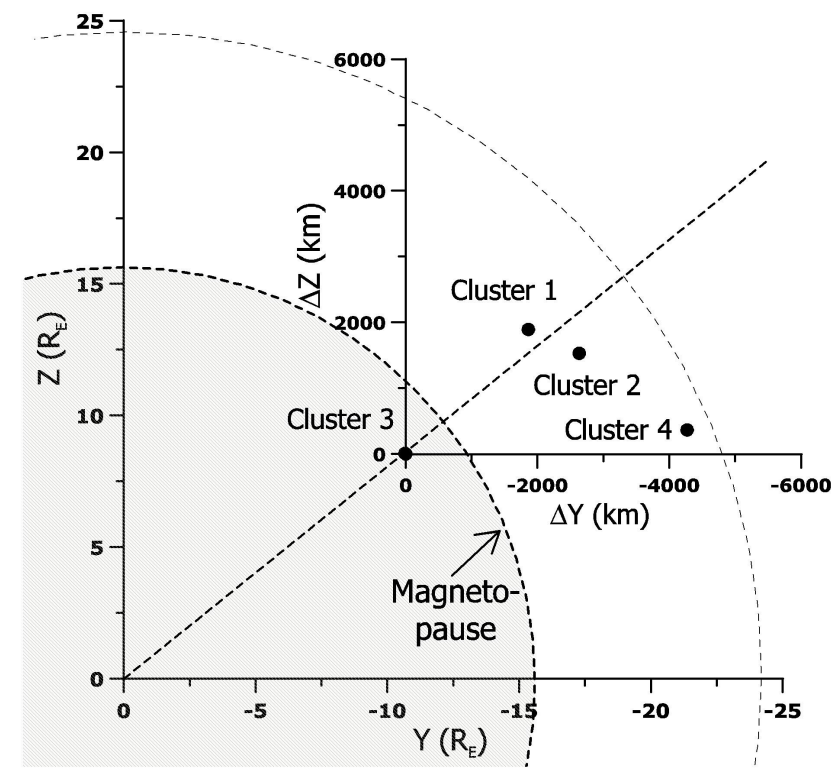

Fig. 7. The Cluster 3 position projected over the YZ plane, and the Cluster 1,2 and 4 positions as pairs of $(\Delta Y, \Delta Z)$ in reference to Cluster 3 are shown. The ideally circular magnetopause surface is drawn with thick-dashed line and encloses the Cluster 3 site throughout the studied event, except for the short intervals that coincide with the occurrence times of the three solar wind pressure pulses.

netopause surface oscillates, while the magnetic field component $\mathrm{B}_{y}$ of Cluster 3 (first subinterval; Fig. 5) and Cluster 2 (second subinterval; Fig. 8) is directly associated with the spacecraft' entries into, and exits out of, the magnetosphere. In both subintervals the magnetopause oscillates and actually constitutes a broad-band energy source that is able to propagate inward fast mode waves.

The Cluster $2 \mathrm{~B}_{y}$ line at $\mathrm{T} \cong 3.5 \mathrm{~min}$ (Fig. 11) may excite the FLR, which is also visible along the trace of the BJN PSD (Fig. 11, bottom panel). The same line of $\mathrm{T} \cong 3.5 \mathrm{~min}$, for the BJN station, is strong during the first subinterval (Fig. 10, fourth panel) as well, in which a second line with $\mathrm{T} \cong 7 \mathrm{~min}$ (Fig. 10) is simultaneously apparent. The conjugate point for the BJN station over the $\mathrm{XY}$ plane, as it is determined using the T96 model, is $(\mathrm{X}, \mathrm{Y})=(1.64,-9.7) \mathrm{R}_{E}$. This point corresponds to $\mathrm{L}=9.84$, and given that the POLAR at $\mathrm{L}=9.4$ is associated with the FLR period of $6 \mathrm{~min}$, then it is plausible to hypothesize that the $\mathrm{BJN}$ line of $\mathrm{T} \cong 7 \mathrm{~min}$ is probably the local resonant frequency that corresponds to the fundamental mode oscillation, while the line at $\sim 3.5$ min corresponds to the first harmonic.

\subsection{The cavity/waveguide mode is not revealed}

The discrete and stable frequencies with $\mathrm{T} \cong 2.5$ and $6 \mathrm{~min}$, which are locally observed by LANL 97A and POLAR, respectively, as well as the periods of $\mathrm{T}=3.5$ and $7 \mathrm{~min}$ detected by the BJN ground station, are probably produced by spatially restricted FLRs, and consequently, they are not the 




Fig. 8. From top to bottom: Geotail magnetic field amplitude and solar wind ion pressure shifted in time 13 min to match the POLAR and Cluster data; POLAR ion density profile, and the $\mathrm{B}_{y}$ component of the magnetic field for each of the four Cluster satellites. During this interval Cluster 3 remains inside the magnetopause $\left(\mathrm{B}_{y}>0\right)$, whereas the other three Cluster satellites remain in magnetosheath $\left(\mathrm{B}_{y}<0\right)$ and enter into the magnetosphere proper only for four short intervals. For the same interval the POLAR ion density shows five complete variation cycles. 


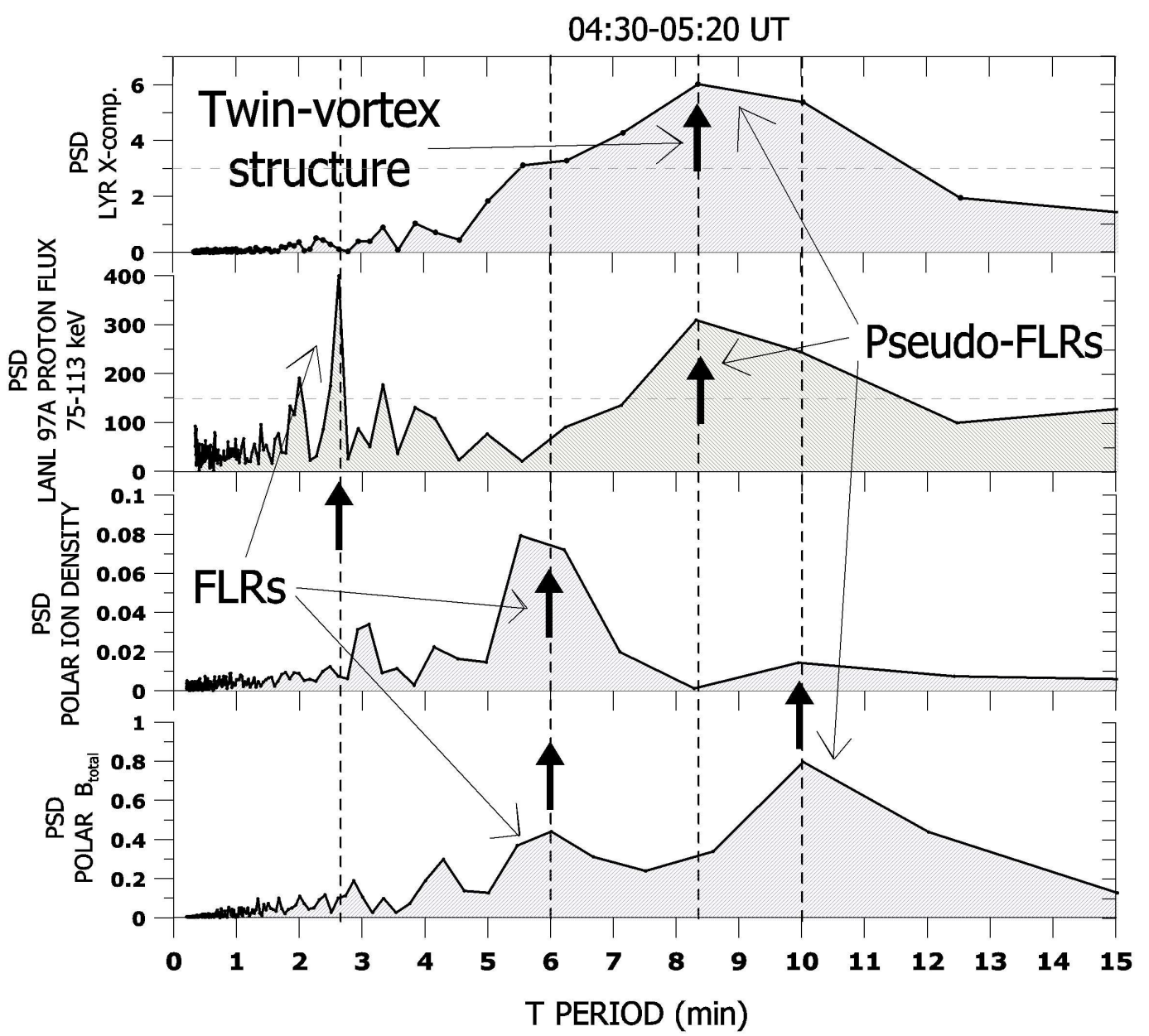

Fig. 9. Power spectral densities (PSDs) of the interval 04:30-05:20 UT for (a) the X-component magnetogram of LYR, (b) the 75-113 keV proton differential fluxes of LANL 97A, (c) the POLAR ion density, and (d) the POLAR magnetic field magnitude. The almost monochromatic lines of $\mathrm{T}=2.5$ and $6 \mathrm{~min}$ correspond to FLRs, whereas the broad-band response of LYR is associated with the detected twin vortex ionosphere structures, which are produced by the solar wind pressure pulses.

eigenfrequencies developed within the magnetosphere from a supposed cavity/waveguide mode. The latter is obvious because if we had adopted the cavity mode scenario, then the lines of $\mathrm{T}=2.5$ and 6 min should have been observed by both satellites (i.e. POLAR and 97A). Conversely, they are developed and observed locally. During the interval presented in Fig. 6, 04:30-05:20 UT, the LYR station shows a main frequency at $\mathrm{T} \cong 8.5 \mathrm{~min}$ (Fig. 9), however, the wave activity has a more broad-band character that extends from $\mathrm{T} \cong 5.5$ to $12 \mathrm{~min}$. Therefore, by itself, the ground response is not monochromatic, as the cavity mode model requires. We mention, again, that the cavity mode model was introduced to reconcile the development of monochromatic pulsations observed on the ground with a broad-band energy input via the KHI. The PSD of LYR (Fig. 9) probably reflects the broadband character seen in the solar wind variation parameters that directly affect the magnetosphere. Actually, apart from the unambiguously recognized FLR frequencies, the POLAR and 97A measurements show a wave activity that is due to the solar wind conditions rather than to the cavity mode. As a matter of fact, only the time series dependent on the total magnetic field B are affected within the magnetosphere. The whole magnetosphere is successively compressed and decompressed, the magnetic field amplitude is directly affected (the POLAR case, Fig. 6) and, in turn, the energetic particle fluxes at the 97A site are appropriately modulated. The POLAR ion density remains intact by the magnetic field magnitude microvariations, while the plasma vortices are essentially associated with the toroidal magnetic field oscillations.

The magnetopause surface constitutes a rather broad-band source of waves. The $\mathrm{B}_{y}$ trace of Cluster provides the appropriate parameter associated with the motion of the magnetopause boundary and its structure; and the $\mathrm{B}_{y}$ PSD may correspond to the imparted energy across the boundary. Therefore, the periods included in the band $\mathrm{T}=8-11 \mathrm{~min}$ (Figs. 10 and 11, for the Cluster $\mathrm{B}_{y}$ PSDs) probably force the magnetosphere to oscillate, whereas a cavity/waveguide mode is not developed. Under this perspective, the LYR and BJN station responses, with maximum PSDs at $\mathrm{T} \cong 8.3$ and $10 \mathrm{~min}$, 


\section{First Interval}



Fig. 10. For the "first interval" 04:20-05:10 UT the PSDs are computed for (a) the Geotail solar wind ion pressure, (b) the Geotail magnetic field amplitude upstream of the bow shock, (b) the Cluster $3 \mathrm{~B}_{y}$ magnetic field component, and (c) the X-component magnetogram from the BJN station. The Geotail data are shifted 13 min to match the Cluster measurements. The BJN station shows one peak PSD corresponding to the twin vortex structures (like the other ground stations), however, two distinct resonant frequencies with $\mathrm{T}=3.5$ and $7 \mathrm{~min}$ are also evident.

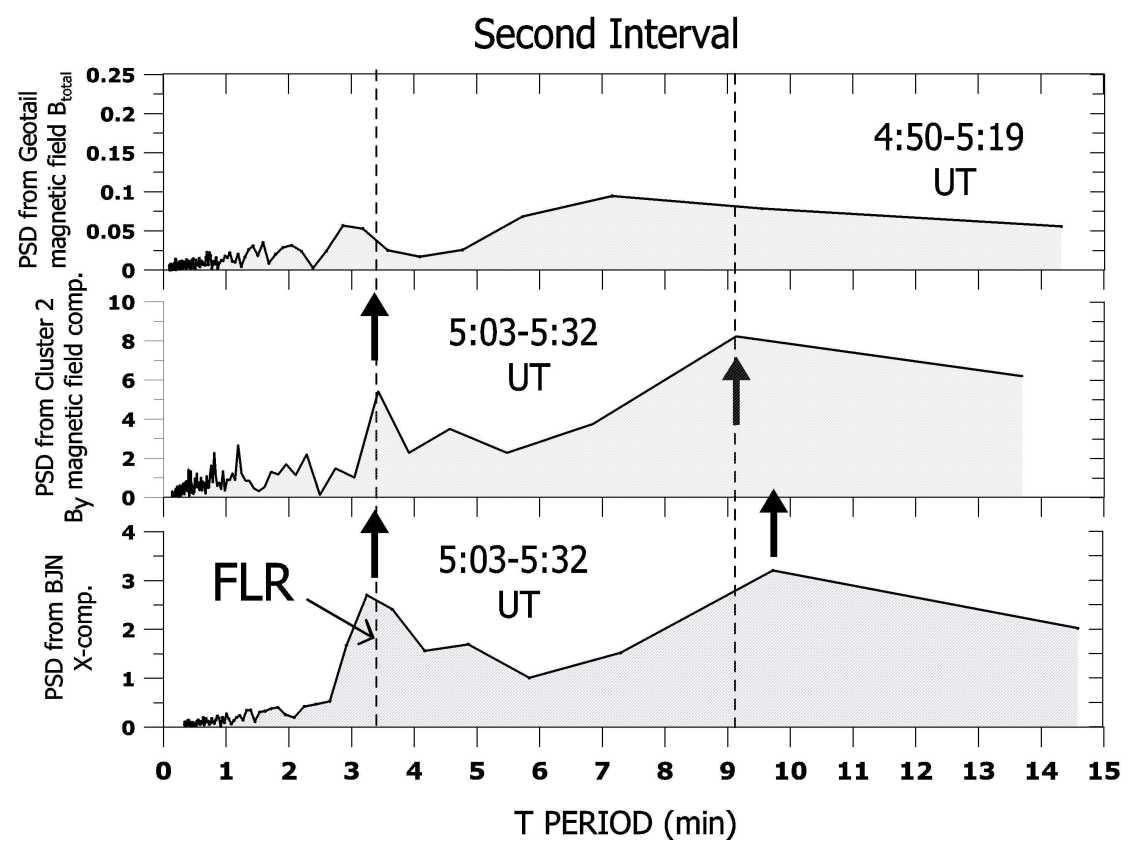

Fig. 11. For the "second interval" 05:03-05:32 UT the PSDs are presented with the same format as in Fig. 10. The BJN station shows the resonant frequency of $\mathrm{T}=3.5 \mathrm{~min}$, too. 
respectively, as well as the 97A proton flux response with $\mathrm{T} \cong 8.3 \mathrm{~min}$, are forced oscillations. The magnetosphere compressional waves, in this case, mimics the behavior of the supposed global cavity/waveguide modes, but their origin is not the fast-mode wave energy trapped in the magnetosphere. The "forced oscillations" imposed by the exo-magnetosphere pressure pulses are manifested over the ionosphere as twin vortex systems.

The event under study in this article could be considered as an ideal case for excited and observed cavity mode oscillations. The magnetosphere is successively compressed, the magnetopause boundary actually oscillates quasi-periodically, and fast mode waves probably propagate within the magnetosphere cavity. However, we fail to recognize any element that might support the cavity mode model.

\subsection{The cavity mode model of Samson}

The cavity mode model of Samson (CMS) is associated with the discrete (monochromatic) lines at $\mathrm{T}=4,4.9,6.4,8.8$ and $12.8 \mathrm{~min}$. Our intention is to scrutinize each line in the light of the presented case study, and in particular, to study the first subinterval which is directly associated with the three intense solar wind pressure pulses. Apparently, the CMS lines of 4, 4.9, and $12.8 \mathrm{~min}$ are not observed, neither by satellite nor by ground stations. The CMS line of $6.4 \mathrm{~min}$ may agree with the POLAR magnetic field and ion density oscillation frequency, but the LANL 97A satellite, at the same time, does not trace such a line. The CMS line of $8.8 \mathrm{~min}$ is not observed in the POLAR magnetic field and the BJN $\mathrm{X}$-component spectra, which show a peak PSD of $\mathrm{T}=10 \mathrm{~min}$. Most importantly, the line of $8.8 \mathrm{~min}$ is within the frequency band extended from 8 to $10 \mathrm{~min}$, corresponding to the formation of the twin vortex structures, the so-called pseudo-FLRs (Sarafopoulos, 2005). The latter are associated with the dispersive structures of ground magnetograms shown, for the case under study, by Sarafopoulos (2004b, Fig. 1). Actually, the LYR station PSD is peaked in between $\mathrm{T}=8$ and $10 \mathrm{~min}$ (Fig. 9, top panel); and the same result is inferred from the Cluster $3 \mathrm{~B}_{y}$ PSD (Fig. 10, third panel), which reflects the magnetopause dynamics. Therefore, our crucial conclusion is that no CMS frequency is detected in this case study.

\section{Conclusion}

Despite the well-organized multipoint observations, no evidence of any eigenfrequency associated with a cavity mode oscillation is detected within the magnetosphere. Our final opinion is that we have to look at a few more similar case studies with multipoint observations. If the additional observational evidence aligns with this study result, then we will have to abandon the idea that the magnetosphere resonates as a cavity within the Pc5 frequency band.

Acknowledgements. We are grateful to all Principal Investigators of the experiments MGF and CPI/SWA of Geotail; TIDE, MFE and EFI of POLAR; MAG and SWEPAM of ACE; FGM of Cluster and the energetic particle experiment of LANL 97A. Concerning the use of ground magnetometer data, we are grateful to the Finnish Meteorological Institute (FMI/GEO), the Technical University of Braunschweig and other institutes that maintain the IMAGE magnetometer array. We also thank both of referees.

Topical Editor T. Pulkkinen thanks K. Takahashi and K.-H. Glassmeier for their help in evaluating this paper.

\section{References}

Allan, W., White, S. P., and Poulter, E. M.: Impulse-excited hydromagnetic cavity and field-line resonances in the magnetosphere, Planet. Space Sci., 34, 371-385, 1986.

Anderson, B. J., Engebretson, M. J., Rounds, S. P., Zanetti, L. J., and Potemra, T. A.: A statistical study of Pc 3-5 pulsations observed by the AMPTE/CCE magnetic fields experiment, 1, Occurrence distributions, J. Geophys. Res., 95, 10 495-10 523, 1990.

Baker, Gregory J., Donovan, E. F., and Jackel, Brian J.: A comprehensive survey of auroral latitude Pc5 pulsation characteristics, J. Geophys. Res, 108A10, 1384, doi:10.1029/2002JA009801, 2003.

Balogh, A., Dunlop, M. W., Cowley, S. W. H., Southwood, D. J., and Thomlinson, J. G., and the Cluster magnetometer team: The Cluster Magnetic Field Investigation, Space Sci. Rev., 79, 65-92, 1997.

Chen, L. and Hasegawa, A.: A theory of longperiod magnetic pulsations 1 . Steady state excitation of field line resonance, J. Geophys. Res., 79, 1024-1032, 1974.

Chisham, G. and Orr, D.: A statistical study of the local time asymmetry of Pc5 ULF wave characteristics observed at midlatitudes by SAMNET, J. Geophys. Res., 102(A11), 24 339-24350, 1997.

Engebretson, M. J., Zanetti, L. J., Potemra, T. A., and Acuna, M. H.: Harmonically structured ULF pulsations observed by the AMPTE CCE magnetic field experiment, Geophys. Res. Lett., 13, 905-908, 1986.

Fenrich, F. R., Samson, J. C., Softko, G., and Greenwald, R. A.: ULF high- and low- $m$ field line resonances observed with the Super Dual Auroral Radar Network, J. Geophys. Res., 100(A11), 21 535-21 547, 1995.

Francia, P. and Villante, U.: Some evidence of ground power enhancements at frequencies of global magnetospheric modes at low latitudes, Ann. Geophys., 15, 17-23, 1997,

SRef-ID: 1432-0576/ag/1997-15-17.

Harrold, B. G. and Samson, J. C.: Standing ULF modes of the magnetosphere: a theory, Geophys. Res. Lett., 19, 1811-1814, 1992.

Hughes, W. J.: Magnetospheric ULF waves: A tutorial with a historical perspective, in Solar Wind Sources of Magnetospheric ULF Waves, Geophysical Monogr. Ser., vol. 81, (Eds.) Engebretson, M. J., Takahashi, K., and Scholer, M., 1-11, AGU, Washington, D.C., 1994.

Glassmeier, K. H.: ULF pulsations, in Handbook of Atmospheric Electrodynamics, Volume II, (Ed.) Volland, H., 463-502, University of Bonn, Germany, 1995a.

Glassmeier, K. H.: Ultralow-frequency pulsations: Earth and Jupiter compared, Adv. Space Res., 16, 4, 4209-4218, 1995 b.

Glassmeier, K. H., Othmer, C., Cramm, R., Stellmacher, M., and Engebretson, M.: Magnetospheric field line resonances: A comparative planetology approach, Surveys in Geophys. 20, 61-109, 1999. 
Kivelson, M. G., Etcheto, J., and Trotignon, J. G.: Global compressional oscillations of the terrestrial magnetosphere: the evidence and a model, J. Geophys. Res., 89, 9851-9856, 1984.

Kivelson, M. G. and Southwood, D. J.: Resonant ULF waves: A new interpretation, Geophys. Res. Lett., 12, 49-52, 1985.

Kivelson, M. G. and Southwood, D. J.: Coupling of global magnetospheric MHD eigenmodes to field line resonances, J. Geophys. Res., 91, 4345-4351, 1986.

Korotova, G. I. and Sibeck, D. G.: A case study of transient event motion in the magnetosphere and in the ionosphere, J. Geophys. Res., 100, 35-46, 1995.

Lee, D.-H. and Lysak, R. L.: Impulsive excitation of ULF waves in the three-dimensional dipole model: the initial results, J. Geophys. Res., 96, 3479-3486, 1991a.

Lee, D.-H. and Lysak, R. L.: Monochromatic ULF wave excitation in the dipole magnetosphere, J. Geophys. Res., 96, 5811-5817, $1991 b$

Lee, D.-H. and Lysak, R. L.: Magnetospheric ULF wave coupling in the dipole field: the impulsive excitation, J. Geophys. Res., 94, 17 097-17 103, 1989.

Mathie R. A., Mann I. R., Menk F. W., and Orr D.: Pc5 ULF pulsations associated with waveguide modes observed with the IMAGE magnetometer array, J. Geophys. Res., 104, 7025-7036, 1999.

Matsuoka, H., Takahashi, K., Yumoto, K., Anderson, B. J., and Sibeck, D. G.: Observation and modeling of compressional Pi 3 magnetic pulsations, J. Geophys. Res., 100, 12 103-12 115, 1995.

McDiarmid, D. R. and Allan, W.: Simulation and analysis of auroral radar signatures generated by a magnetospheric cavity mode, J. Geophys. Res., 95(A12), 20 911-20 922, 1990.

Mitchell, D. G., Engebretson, M. J., Williams, D. J., Cattell, C. A., and Lundin, R.: Pc5 Pulsations in the outer dawn magnetosphere seen by ISEE 1 and 2, J. Geophys. Res., 95, 967-975, 1990.

Nosé, M., Iyemori, T., Sugiura, M., and Slavin, J. A.: A strong dawn/dusk asymmetry in Pc 5 pulsation occurrence observed by the DE-1 satellite, Geophys. Res. Lett., 22, 2053-2056, 1995.

Provan, G. and Yeoman, T. K.: A comparison of field-line resonances observed at the Goose Bay and Wick radars, Ann. Geophys., 15, 231-235, 1997, SRef-ID: 1432-0576/ag/1997-15-231.

Samson, J. C., Harrold, B. G., Ruohoniem, J. M., Greenwald, R. A., and Walker, A. D. M.: Field line resonances associated with MHD waveguides in the magnetosphere, Geophys. Res. Lett., 19, 441-444, 1992.
Samson, J. C., Greenwald, R. A., Ruohoniemi, J. M., Hughes, T. J., and Wallis, D. D.: Magnetometer and radar observations of magnetohydrodynamic cavity modes in the earth's magnetosphere, Can. J. Phys., 69, 929-937, 1991.

Sarafopoulos, D. V.: Long duration Pc 5 compressional pulsations inside the Earth's magnetotail lobes, Ann. Geophys., 13, 926937, 1995

SRef-ID: 1432-0576/ag/1995-13-926.

Sarafopoulos, D. V.: Distinct solar wind pressure pulses producing convection twin-vortex systems in the ionosphere, Ann. Geophys., 22, 2201-2211, 2004a,

SRef-ID: 1432-0576/ag/2004-22-2201.

Sarafopoulos, D. V.: Repetitive X-line Hall current structures over the dawnside ionosphere induced by successive exomagnetosphere pressure pulses, Ann. Geophys., 22, 4153-4163, 2004b,

SRef-ID: 1432-0576/ag/2004-22-4153.

Sarafopoulos, D. V.: Pseudo-Field Line Resonances in ground Pc5 pulsation events, Ann. Geophys., 23, 593-608, 2005, SRef-ID: 1432-0576/ag/2005-23-593.

Shimazu, H., Araki, T., Kamei, T., and Hanado, H.: A symmetric appearance of Pc 5 on dawn and dusk side associated with solar wind dynamic pressure enhancement, J. Geomag. Geoelectr., 47, 177-189, 1995.

Southwood, D. J.: Some features of field line resonances in the magnetosphere, Planet. Space Sci., 22, 483-491, 1974.

Takahashi, K.: ULF waves: 1997 IAGA division 3 reporter review, Ann. Geophys., 16, 787-803, 1998,

SRef-ID: 1432-0576/ag/1998-16-787.

Tsyganenko, N. A.: Modelling the Earth's magnetospheric magnetic field confined within a realistic magnetopause, J. Geophys. Res., 100, 5599-5612, 1995.

Tsyganenko, N. A.: Effects of the solar wind conditions on the global magnetospheric configuration as deduced from data-based field models, European Space Agency Spec. Publ., ESA SP-389, 181,1996

Walker, A. D. M., Ruohouniemi, J. M., Baker, K. B., Greenwald, R. A., and Samson, J. C.: Spatial and temporal behaviour of ULF pulsations observed by the Goose Bay HF radar, J. Geophys. Res., 97, 12 187-12 202, 1992.

Ziesolleck, C. W. S. and McDiarmid, D. R.: Statistical survey of auroral latitude Pc 5 spectral and polarization characteristics, J. Geophys. Res., 100, 19299-19312, 1995. 